
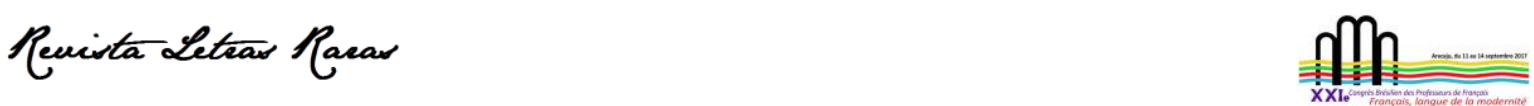

Actes du XXlème Congrès Brésilien des Professeurs De Français dans Édition spéciale de la Revue Letras Raras, 2021 ISSN : 2317-2347 - v. 10, Dossier Spécial (2021)

Todo o conteúdo da RLR está licenciado sob Creative Commons Atribuição 4.0 Internacional

\title{
Actes du XXle Congrès Brésilien des Professeurs de Français
}

Que le temps passe vite, on ne doute pas. Cela fait déjà quatre ans que la ville d'Aracaju, située sur la côte du Nordeste, a accueilli le XXle Congrès brésilien des professeurs de français, organisé ${ }^{1}$ par la Fédération Brésilienne des Professeurs de Français et l'Association des Professeurs de Français de l'État de Sergipe (renommée depuis AFRASE). Mais les belles rencontres laissent toujours leurs empreintes dans nos mémoires à travers tout ce que l'on y apprend en partage.

Du 11 au 14 septembre 2017 nous étions réuni.e.s autour du thème « Français, langue de la modernité : une voie vers des cultures et des savoirs multiples ». Nous étions 368 participant.e.s : enseignant.e.s, chercheurs et chercheuses, étudiant.e.s venu.e.s de toutes les régions du Brésil, mais aussi de France, de Belgique, du Québéc, d'Argentine, du Paraguay, d'Uruguay, du Mexique et de Roumanie. Nous avons partagé nos expériences professionnelles, nos parcours de formation, nos savoirs, nos savoir-faire, nos savoir-enseigner et apprendre. Les travaux ont été regroupés sur 4 axes thématiques (1- Didactique et formation de professeurs ; 2Littérature et arts ; 3-Politique et mémoire ; 4- Études de traduction et études linguistiques) ${ }^{2}$ et ont été présentés sous la forme de conférence, table ronde, communication, atelier, affiche et exposition photo sur une expérience pédagogique. Le programme a été enrichi par des activités culturelles et des lancements de livres.

Même si les échos positifs ont traversé ces quatre années, jusqu'à l'année dernière nous n'avions pas eu l'occasion de publier les textes. Alors, en 2020 nous avons décidé de donner le cahier de résumés complet dans une édition spéciale de la Revista Letras Raras, qui nous a gentiment offert ses pages, par l'intermédiaire de la FBPF. Nous renouvelons, maintenant, cette

\footnotetext{
${ }^{1}$ Avec le soutien de l'Ambassade de France au Brésil, du Consulat Général de France à Recife, de l'Université Fédérale de Sergipe, du Collège d'Application de l'UFS, de l'Alliance Française d'Aracaju, de la Délégation Générale des Alliances Françaises du Brésil, de la Fédération Internationale des Professeurs de Français, du Secrétariat d'État à la Culture de Sergipe, de CLE Internationale, du Français dans le Monde, de TV5MONDE, de Radio France Internationale - RFI, de Hachette - Langue Étrangère, des Éditions Didier, de Bonjour de France, des Universités du Monde, du Centre de Formation pour Apprentis la Noue - Bourgogne et de Siga Dance. Un grand merci à tous ces partenaires qui ont rendu possible notre Congrès, ainsi qu'à toute l'équipe d'organisation et les participant.e.s.

2 Le Comité Scientifique était composé par les professeur.e.s : Christianne Benatti Rochebois (UFSB); Cristina Casadei Pietraróia (USP) ; Dario Pagel (UFS) ; Joice Armani Galli (UFPE, à l'époque) ; Josilene Pinheiro-Mariz (UFCG) ; Kátia Ferreira Fraga (UFPB) ; Maria Elizabeth Chaves de Mello (UFF); Marie-Hélène Torres (UFSC) ; Márcio Venício Barbosa (UFRN) ; Renilson Santos Oliveira (UFS) ; Telma Cristina Pereira (UFF) et Wellington Júnio Costa (UFS). Un grand merci à ces collègues si compétent.e.s.
} 

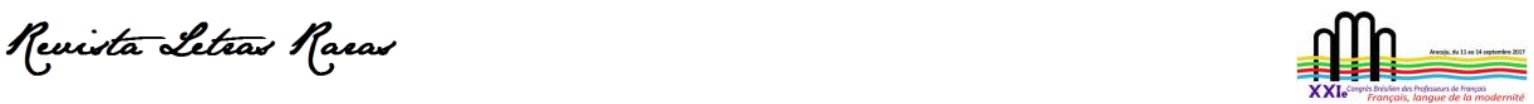

Actes du XXlème Congrès Brésilien des Professeurs De Français dans Édition spéciale de la Revue Letras Raras, 2021 ISSN : 2317-2347 - v. 10, Dossier Spécial (2021)

Todo o conteúdo da RLR está licenciado sob Creative Commons Atribuição 4.0 Internacional

collaboration réussie avec la publication de ces Actes. Suite à un appel à contribution, nous avons reçu des textes représentatifs des quatre axes du congrès, que nous reproduisons dans l'ordre suivant :

D'abord, les mots de la conférence donnée par Jean-Louis Chiss (Sorbonne Nouvelle DILTEC), qui rend hommage à la mémoire de la professeure Sabina Kundman et nous présente sous le titre "Le français (langue et littérature) et l'idée de "modernité" » une réflexion sur la thématique du congrès, à travers laquelle l'auteur explore la relation entre la langue française et le concept de modernité « dont on sait l'inépuisable polysémie et le jeu de renvois, de croisements, de métamorphoses qu'il suscite ».

Ensuite, nous pouvons lire dans « Le professeur de DdNL et sa formation », de Fernanda Cristina Puça França (Cap - UFPE) une description détaillée et réfléchie des caractéristiques de l'enseignement d'une discipline dite Non Linguistique en langue française, du rôle du professeur responsable pour ce travail et de sa formation. Pedro Armando de Almeida Magalhães (UERJ), pour sa part, nous propose une réflexion sur l'enseignement du français de spécialité et du français sur objectif(s) spécifique(s). Ciblant le Français du Droit, l'auteur de « Systèmes juridiques français et brésilien: enjeux de la conception des cours français du droit » nous montre les différences les plus importantes entre le système juridique français et le système juridique brésilien, tout en relevant les défis qui en découlent pour la conception du cours Français du Droit. Cláudia OzonCaldo (UFTPR), dans son texte « Quelles perspectives pour les cours de français aux Centres de Langues Universitaires? », nous présente des perspectives pour l'enseignement du Français Langue Étrangère (FLE) dans les centres universitaires de langues, à partir de son expérience auprès des étudiants en génie, entre autres, à l'Université Fédérale Technologique du Paraná. Avec «L'évaluation formative en français langue étrangère : le portfolio et l'écriture réflexive en contexte universitaire », Denise Gisele de Britto Damasco nous invite à réfléchir au sujet de l'évaluation formative en rapportant son expérience didactique menée en 2016 à l'Institut de Lettres d'une université publique brésilienne avec des futurs enseignants de français, des professionnels en Langues Etrangères Appliquées et des traducteurs.

Pour ce qui est de l'axe littéraire, nous avons dans ces Actes deux des travaux présentés au congrès. Le premier, "Littératures dans l'enseignement du Français Langue Étrangère : réflexion et pratique à I'UFPR », de Viviane Araújo Alves da Costa Pereira (UFPR) et Cláudia Helena Daher (UFPR), est le rapport d'un projet d'extension intitulé Littératures dans 

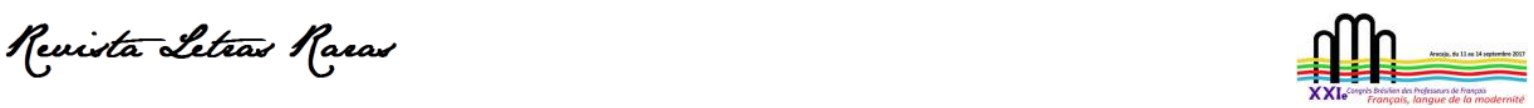

Actes du XXlème Congrès Brésilien des Professeurs De Français dans Édition spéciale de la Revue Letras Raras, 2021 ISSN : 2317-2347 - v. 10, Dossier Spécial (2021)

Todo o conteúdo da RLR está licenciado sob Creative Commons Atribuição 4.0 Internacional

l'enseignement du Français Langue Étrangère (LIFLE) et développé par les deux auteures à l'Université Fédérale du Paraná depuis novembre 2016. II s'agit d'un projet interdisciplinaire qui vise à mettre en œuvre la lecture de textes littéraires, avec le double but de discuter de la place de la littérature en classe de FLE et de diffuser la littérature contemporaine en français. Le second, présenté par Karol Souza Garcia (UFC) et Robert Ponge (UFRGS), a pour titre «Éléments d'analyse du personnage de Lucien dans "L'enfance d'un chef" de Jean-Paul Sartre ». Garcia et Ponge se penchent sur « L'Enfance d'un chef », nouvelle publiée dans Le Mur (1939), recueil de Jean-Paul Sartre, pour analyser la formation du protagoniste, son cheminement pour devenir un chef, tout en soulignant la manière dont ce récit de formation se situe dans son temps, dialogue avec son époque à travers l'utilisation de l'ironie qui met en question les choix du personnage Lucien.

Le volet politique est ici représenté par deux travaux : celui de Christianne Benatti Rochebois (UFSB) et celui, collectif, de Denise Gisele de Britto Damasco (FBPF), Pedro Armando de Almeida Magalhães (UERJ), Nathalie Ceulemans (PROFFF) et Heloisa Caldeira Alves Moreira (APFESP). Alors que dans le premier, «L'enseignement des langues en quête d'une perspective sur l'égalité de chances », Rochebois aborde les politiques éducatives et les capacités des acteurs de l'éducation et fait quelques propositions didactiques autour de l'articulation entre l'éducation inclusive et l'enseignement/apprentissage du français; le deuxième, intitulé « La vie associative : vision, défis et nouveaux projets ", est un rapport de la table ronde qui avait réuni dans le cadre de notre congrès la présidente de l'Association des Professeurs de Français du District Fédéral, le président de l'Association des Professeurs de Français de l'État de Rio de Janeiro, la viceprésidente de l'Association des Professeurs de Français en Flandres - Belgique et la présidente de l'Association des Professeurs de Français de l'État de São Paulo.

Le quatrième et dernier axe thématique du congrès portait sur les études de traduction et linguistiques. Ici, nous publions le travail de Vinícius Stangherlin (UFRGS) et Robert Ponge (UFRGS) intitulé « De la théorie des difficultés de compréhension et/ou de traduction du FLE à l'étude de leurs manifestations : le cas de "Banque" ", dans lequel les auteurs présentent quelques aspects importants des travaux menés à l'Université Fédérale du Rio Grande do Sul dans un laboratoire de recherche sur les difficultés de compréhension et/ou de traduction du français langue étrangère, avec un focus sur l'homonymie, la polysémie, la paronymie et les faux amis. 
Actes du XXlème Congrès Brésilien des Professeurs De Français dans Édition spéciale de la Revue Letras Raras, 2021 ISSN : 2317-2347 - v. 10, Dossier Spécial (2021)

Todo o conteúdo da RLR está licenciado sob Creative Commons Atribuição 4.0 Internacional

Nous ne pourrions finir cette présentation sans exprimer notre sincère remerciement à toute l'équipe éditoriale de la Revista Letras Raras, spécialement à Maria Rennally Soares da Silva (UEPB) et à Josilene Pinheiro-Mariz (UFCG). Nous remercions également l'Ambassade de France au Brésil pour le soutien et notre chère présidente de la Fédération Brésilienne des Professerus de Français, Denise Gisele de Britto Damasco, tout comme le comité de lecture de ces Actes et, bien sûr, les auteur.e.s.

Pour conclure, chères lectrices, chers lecteurs, nous vous souhaitons un très bon moment d'apprentissage et de plaisir. 